\title{
1 Multiscale analysis of a non-periodic epidemic time series using wavelet
}

\section{2 transform}

3 Jean Gaudart $^{*}$, Stanislas Rebaudet ${ }^{2}$, Gaetan Texier $^{3}$, Robert Barrais ${ }^{4}$, Renaud Piarroux ${ }^{5}$ And

4 Roch Giorgi ${ }^{1}$.

5 1: Aix Marseille Univ, APHM, INSERM, IRD, SESSTIM, Hop Timone, BioSTIC, Biostatistic

6 \& ICT, Marseille, France.

7 2: APHM, Hop Timone ; Hôpital Européen Marseille, France.

8 3: Centre for Epidemiology and Public Health, French Forces Health Services, Marseille, 9 France.

10 4: Ministère de la Santé Publique et de la Population, Port-au-Prince, Haiti.

115 : Sorbonne Université, INSERM, Institut Pierre-Louis d'Epidémiologie et de Santé Publique, 12 AP-HP, Hôpital Pitié-Salpêtrière, France.

$13 *$ corresponding author: jean.gaudart@univ-amu.fr

\section{Summary}

The aim of the present study was to develop a method for multiscale analysis of non-stationary and non-periodic epidemic time series. Indeed, the epidemiologists may need to know the features, at different resolutions, of short duration outbreaks that did not exhibit periodic cycles. Among of the large number of wavelets, we have developed a continuous wavelet that shows an analogous shape to the Haar wavelet, and leads to precise time localization. We applied the wavelet transform to the cholera epidemic, which began in October 2010 in Haiti. We determined the wavelet spectra of both the cholera case toll and rainfall time series, from September 01, 2010, to November 20, 2012 (812 days). The relationship between case toll and rainfall was analyzed using cross-wavelet spectra at different lags. Cholera case toll scalogram highlighted four epidemic bursts. Cross-wavelet analysis highlighted an absence of relationship 
between the first epidemic burst and rainfall, but a clear relationship between the following epidemic bursts and rainfall after a 3 to 8 day lag.

Keywords: non-periodic series, non-stationary series, spectral analysis, time series, epidemic, wavelet transform.

\section{Introduction}

Wavelet transform has been more and more applied as an effective tool for non-stationary time series analysis when Fourier analysis cannot be used in order to extract the main characteristics of such series [1,2]. These characteristics include periodic variations, regime shifts or sudden perturbations and gaps. Wavelet transform has been used as a signal processing tool in different domains such as climatology [3,4,5], geophysics [6], neurology [7] or image processing [8,9], and for statistical estimation methods [10,11]. More recently, it has been used in epidemiology for analyzing long time series of cases, and as assessing relationships between case time series and factor time series [12-16]. A complete description of wavelet transform analysis can be found in Torrence and Compo [17] and in Nason [18].

Classically, the studied time series are long-term observations (ten years, or more), and present periodic variations that are studied using periodic wavelets. The main information that can be extracted by such analyses are: i) the frequency analysis, assessing periodicity and periodic changes, ii) the time analysis, assessing variations of time and speed, iii) the comparison of two time series (e.g. epidemic and climatic series [19]). Wavelet transform analysis is based on the choice of one of the different wavelet functions [20]. This choice is based on the observed characteristics of the time series. Classically, two types of wavelet can be used. The Morlet wavelet function [21] is very useful for frequency analyses of periodic time series [22]. Conversely, the Haar wavelet function is more accurate for time analyses of non-periodic nonstationary time series that exhibit rapid changes [17]. Indeed, frequency and time are related by the uncertainty principle [23]. A wavelet function with accurate frequency localization (such 
as the Morlet function) can precisely assess frequencies of a periodic time series, but does not present an accurate time localization, leading to less precise analyses of rapid changes. In the case of a recent emergence of a pathogen in a given territory, the lack of historical data precludes from analyzing periodicity (frequency analysis) and leads to analyze time of changes (time analysis).

The Haitian cholera epidemic that began in October 16, 2010 [24] rapidly proved to be the world's largest epidemic during the Seventh Pandemic [25]. Cholera is an infectious disease due to toxigenic Vibrio cholera $\mathrm{O} 1$, a bacteria that can be directly transmitted from human to human or via environment. As cholera was imported probably for the first time in Haiti, no historical data could be used to assess periodicity (such as seasonality) even after 2 years. Nevertheless, this short-term time series presented rapid variations that could be explained as different regime shifts or epidemic processes. To accurately extract these times of changes in the epidemic process, a periodic wavelet function (such as Morlet function) was thus considered less useful than a time localized wavelet, such as the Haar function. Yet, the Haar function presents discontinuities that lead to discretization problems [26]. The aim of this work was therefore to develop a continuous wavelet function [27,28], and apply the corresponding wavelet transform to the non-periodic non-stationary time series of the Haitian cholera epidemic.

The present article is organized as follows: Section 2 provides a description of the wavelet transform principles and a brief revue of the different wavelet analysis methods, which highlights the usefulness of the scale and translation parameters. It describes the main properties that should guide the choice of the most suitable wavelet function (continuous and non-periodic). It also presents the new continuous wavelet transform we have developed to assess the non-periodic Haitian cholera epidemic and its relationship with rainfall. Section 3 presents the results of the wavelet transform applied to the cholera epidemic in Haiti. Wavelet 
spectra of case and rainfall time series were analyzed and cross-wavelet are presented. A comparison to a change point analysis is also presented. Section 4 provides interpretations and discussions of the results.

\section{Method}

\subsection{Signal analysis}

In most cases, the signal analysis process exhibits several phases: the determination of a spectrum, the coding of information, the transmission, and the reconstruction of the signal. For epidemic time series analysis, the whole analysis process is unnecessary: only the determination of spectra at different scales is necessary. Coding, transmission and reconstruction are not useful from an epidemiological point of view. The epidemic records are given as onedimensional time series and these time series are not stationary. These characteristics are useful to choose an appropriate transform.

The Fourier transform is a decomposition of the signal in order to extract the main frequency components. The Fourier transform requires two conditions for the signal. First, the signal must be stationary [7], i.e. with no sudden changes of frequencies and transient periods. Second, it must fit the sum of sinusoids at different frequencies. The Fourier transform does not allow time analysis, and is not applicable in case of sudden frequency variations analysis and scaling change analysis. Moreover, the Fourier integral method considers phenomena in an infinite interval and a spectrum is obtained for the whole signal. This method gives no information on the time localization of a frequency. For all these reasons the Fourier transform cannot be used to analyze a non-periodic non-stationary epidemic time series.

The deficiencies of the Fourier transform in time-frequency analysis were observed by Gabor [23]. In order to study signals having frequencies, which depend on time, the Gabor algorithm provides a localized window function. The signal analysis is performed by a sinusoidal wave of finite duration. The Gabor algorithm defines a time window that divides the sinusoidal wave 
101 into finite parts. Between its starting point and its end, the sinusoidal wave has constant

102 amplitude and frequency, and the window is the product of a Gaussian function by a sinusoidal

103 wave. The Gabor function can be written as:

$104 G(t)=\exp \left\{-\alpha^{2}\left(t-t_{0}\right)^{2}\right\} \exp \{i(2 \pi f t+\varphi)\}$

105 where $\alpha, \mathrm{t}_{0}, \mathrm{f}, \varphi$ are real constants and $i^{2}=-1$.

106 The window, centred at $t_{0}$, analyzes the corresponding section of the signal series. Then, the

107 window is centred at the following value $t_{1}$, closed to the previous one, and so on. An analysis

108 is performed on a first short section of the signal series. Then, the window is shifted and a new

109 analysis is performed. Each section of the signal is analyzed, according to a classical Fourier

110 analysis. The analysis is repeated until the end of the signal series. The frequency of the

111 sinusoidal wave can change when the window is shifted. By introducing a localization

112 parameter $t_{0}$ of the section and a frequency $f_{0}$, the obtained windowed Fourier transform, also

113 called the Gabor transform, measures locally, around time $t_{0}$, the amplitude of the frequency $f_{0}$.

114 The signal analysis is a time-frequency analysis. The window width, and consequently the

115 width of a signal section, is arbitrary, and can thus affect the result of the analysis. With such

116 a windowed transform approach, the Gabor transform can be used to analyze non-stationary

117 time-series. Nevertheless, if frequency variations are very fast and with high amplitudes, then

118 the Gabor transform fails to detect them, because the series has to be stationary within each

119 window. The choice of the window width and this piecewise stationary assumption can bias

120 the Gabor transform of time series with sudden changes. Hence, Gabor transform cannot be

121 used for epidemic analysis either.

122 In order to study signal series showing various scaling properties, Morlet et al. [21] have

123 modified the Gabor function to obtain a wavelet. As defined, for the first time, by Grossmann

124 and Morlet [29], the wavelet transform is a decomposition of a signal by translations and

125 dilations of a single fixed function called the mother wavelet $\Psi$. Wavelet analysis is a time- 
126 frequency analysis of the signal, which permits to estimate the spectral characteristics of the

127 signal as a function of time. During the analysis process, wavelet transform can denoise the

128 signal preserving its main features. The sinusoidal functions of the Fourier transform are

129 replaced by pseudo-periodic functions that decay sufficiently fast, oscillating like waves. The

130 mother wavelet integrates to zero, that is:

$$
\int_{-\infty}^{+\infty} \Psi(t) d t=0
$$

132 The wavelet transform is well adapted to analyze epidemic time series, providing time-

133 frequency analysis at different scales.

\section{$134 \quad 2.2 \quad$ Wavelet properties}

135 Based on a mother wavelet, a wavelet family is obtained by introducing a translation parameter $b$ and a scale parameter $a$, and is given by:

$\Psi\left(\frac{t-b}{a}\right), a, b \in \mathbb{R}, a>0$

138 Where $\mathbb{R}$ denote the set of real numbers.

139 The Morlet complex wavelet family can be written as:

$$
\Psi(t ; a, b)=\exp \left\{i \omega\left(\frac{t-b}{a}\right)\right\} \exp \left\{\frac{(t-b)^{2}}{2 a^{2}}\right\}
$$

141 Where $\omega$ is the angular frequency.

142 The resolution of a wavelet transform $W$ varies with the scale parameter $a$. If the analysing

143 wavelet is narrow, its time localization is precise and accurate, and therefore sudden changes

144 will be detected as well as noise. If the analysing wavelet is wide, the large variations of the

145 signal are exhibited, but the resolution is less accurate and the signal is denoised. The wavelet

146 transform can thus be used as a passband filter, where the bandwidth depends on the value of

147 scale parameter $a$ which defined the analysing window width. The smaller the scale parameter

$148 a$ (narrow analysing wavelet), the more precise and accurate the time resolution. Defining 
presented using scalogram figures. Considering the wavelet $\Psi$ as a real function, the wavelet coefficients $W(a, b)$ of a time series $s(t)$ can be written as:

153 The wavelet coefficients of a time series is defined as the convolution of $s(t)$ and a translated

154 and scaled version of $\Psi(t)$. The time series spectrum is then obtained from $W$, exhibiting the

155 speed of local variations of a time series. To be admissible as a wavelet, a function has to

156 satisfy several crucial properties [29]:

157 Property 1: the function integral converges and is zero mean.

$158 \int_{-\infty}^{+\infty}|\Psi(t)| d t<\infty$ and $\int_{-\infty}^{+\infty} \Psi(t) d t=0$

159 The wavelet values have to divide up, in an equal way, on both sides of the time axis. This

160 implies that $\Psi(t)$ oscillates. When this condition is not verified, the positive values (increase

161 of the time series) or the negative values (decrease of the time series) are privileged to the detriment of the other values.

163 Property 2: the function is a square integrable function.

$164 I=\int_{-\infty}^{+\infty}|\Psi(t)|^{2} d t$

165 Then, the function is normalized such as $\int_{-\infty}^{+\infty}\left|\Psi_{\mathrm{N}}(t)\right|^{2} d t=1$

166 Where $\Psi_{\mathrm{N}}$ is the normalized function. This condition is important for being able to compare the

167 results between each other.

168 Property 3: a function is called an orthogonal wavelet if the following condition is verified:

170 Where $\delta_{i, j}$ is the Konecker symbol. This condition is not obligatory, but if it is satisfied the

171 time series decomposition is unique. The wavelet coefficients are not correlated and the time

172 series is a linear combination of the coefficients. A good reconstruction of the original time 173 series can thus be obtained from the wavelet coefficients. The Daubechies wavelets [30,31] 
174 form an orthogonal basis and allow a good compression of the information, useful for image

175 analysis. Note that the Morlet wavelet does not satisfy the orthogonality condition.

176 Property 4: moments of the wavelet indicate how a function decays toward infinity. Some

177 vanishing moments could verify the following condition:

$178 \int_{-\infty}^{+\infty} \Psi(t) t^{n} d t=0, \quad n \in \mathbb{N}$

179 This condition is not obligatory, but leads to calculation simplifications.

180 Wavelets rarely verify all these properties. It is thus necessary to choose a wavelet which

181 exhibits the properties that are the more useful in the context of the epidemic time series 182 analysis.

\section{$183 \quad 2.3$ Choosing a wavelet for non-periodic series}

184 The choice of a wavelet, for non-stationary time series analysis, is still an open problem. The

185 wavelet function has to reflect the type of features to be extracted. For evident reason, and like

186 many mathematical and statistical approaches, a wavelet must not be chosen only because an

187 algorithm has been developed to perform a wavelet transform. The wavelet function has to be

188 chosen according to the time series to be analyzed, according to the characteristics to be

189 extracted [10,32], and reaching epidemiologic goals (outbreak detection, epidemic mechanism,

190 seasonality, coherence with environmental time series,...).

191 To choose the right wavelet in our study, we first considered the unnecessary wavelet properties and, second, their mandatory properties to assess epidemic onsets. Reconstruction of the

193 original time series from wavelet coefficients was unnecessary. Therefore, the orthogonality

194 condition (property 3) did not need to be satisfied. In studies of epidemiological time series,

195 the Morlet wavelet has been often used [33-35]. But all these series exhibited periodic cycles.

196 In this context, the Morlet wavelet was a good compromise between the determination of the

197 frequencies of these cycles and the time localization of epidemic bursts. However, the Morlet

198 wavelet should be used with caution because it does not verify property 1 , as the mean is not 
null. In our context, studying frequencies was not of interest since we wanted to localize the

epidemic bursts. The uncertainty relation implies that a good accuracy of the frequency values same reason we did not choose the Shannon wavelet [18].

Mandatory properties of wavelets for our study were related to the following. We studied a new cholera epidemic over a relatively short period (about 800 days). Epidemiological series was not smooth and exhibited many sudden transitions of large magnitude. The lack of annual period made it unnecessary to have a wavelet defined on the complex set $\mathbb{C}$, but to use only a real wavelet. Our goal was to precisely date the epidemic bursts. Thus, a wavelet well localized in time was needed to obtain a good resolution. To choose the mother wavelet we kept the following properties:

211 - Properties 1: the wavelet integral must be convergent and zero mean,

213 Moreover, the wavelet had to decay sufficiently fast. The wavelet that best satisfies these 214 properties is the Haar wavelet [18,36]. It is real and precisely localized in time. Its main 215 drawback is that this wavelet is a piecewise-defined function and it is not regular. It presents 216 several significant discontinuities with substantial angularities, which may interfere with the 217 practical computation, especially with a discretization in the vicinity of a discontinuity [26]. 218 Hence, this wavelet may be suitable for the analysis of regular functions but not for sudden 219 changes in time such as epidemic time series. Therefore, we chose a smooth and continuous 220 wavelet, with a shape similar to the Haar wavelet. This chosen mother wavelet, called GCP 221 wavelet, was established by Gaudart et al. [28], and is defined as: 
224 The value of the parameter $c \in \mathbb{R}$ fixes the slope of the wavelet flanks. Depending on the value

225 of this parameter the increase and decrease of the curve is more or less rapid, setting the decay

226 of the wavelet. In the following, $c$ will be fixed to 1 , unless special statement. This value

227 allowed a rapid return to zero, a sufficiently fast decrease of the wavelet, and a precise time

228 localization. Too large values of $c$ led to a slow decay and therefore a poor extraction of sudden

229 changes in the epidemic time series. Small values led to discontinuities.

230 In what follows, the function $\Psi(t)$ represents the GCP wavelet, unless otherwise indicated.

231 This function $\Psi$ is defined on $\mathbb{R}$, convergent, zero mean, and it is a square integrable function.

232 Consequently, the function $\Psi$ is a wavelet. The GCP wavelet family $\Psi(t ; a, b)$ is given by:

$233 \Psi(\mathrm{t} ; \mathrm{a}, \mathrm{b})=\frac{1-\left[\tanh \left(\frac{\mathrm{t}-\mathrm{b}}{\mathrm{a}}\right)\right]^{2}}{\operatorname{coth}(\mathrm{c}) \operatorname{coth}\left(\frac{\mathrm{t}-\mathrm{b}}{\mathrm{a}}\right)-\tanh (\mathrm{c}) \tanh \left(\frac{\mathrm{t}-\mathrm{b}}{\mathrm{a}}\right)}$

234 with $a \in \mathbb{R}^{+*}, b \in \mathbb{R}, c \in \mathbb{R}^{*}$.

235 In order to compare wavelet transforms between each other, the wavelet $\Psi(t ; a, b)$ needs to be 236 normalized [28], following equation (8). The normalized wavelet $\Psi_{\mathrm{N}}(\mathrm{t})$ is written as:

$237 \quad \Psi_{\mathrm{N}}(\mathrm{t} ; \mathrm{a}, \mathrm{b})=\frac{1}{\sqrt{\mathrm{N}}} \Psi(\mathrm{t} ; \mathrm{a}, \mathrm{b})$

238 Where $N$ is the normalized factor such as: $N=\int_{-\infty}^{+\infty}|\Psi(t ; a, b)|^{2} d t$

239 The norm depends on the scale parameter $a$. In the development of the algorithm, the norm $N$

240 thus has to be calculated for each value of the scale parameter. Unless special statement, $\Psi(t)$

241 will be assumed to be the normalized GCP wavelet.

242 In our context, the analysis of the epidemic time series was performed using wavelet translation,

243 whatever the width of the wavelet. The entire epidemiologic time series was scanned by the

244 wavelet, including a period prior the beginning of the cholera epidemic. Therefore, the value

245 of the translation parameter $b$ ranged from 1 to 812 days. Variation of the scale parameter

246 showed different wavelet shapes (figure 1). The upper curve in figure 1 is a narrow wavelet

247 (scale parameter $a=1)$, centred at $t=5$ (translation parameter $b=5)$. The middle curve is a 
wavelet of average width $(a=2)$, centred at $t=7$. The lower curve is a wide wavelet $(a=$

3 ), centred at $t=9$. In order to determine the wavelet width, we defined, for a given time $t$,

250 the ratio: $r=\frac{\Psi(\mathrm{t})}{\Psi_{\max }}$

251 Where $\Psi_{\max }>0$ is the maximal positive value of the wavelet for a given value of the scale

252 parameter. Width of the useful part of a wavelet is given by the condition:

$253-1<r<-0.05$ or $1>r>+0.05$

254 Outside these ranges the values of $\Psi(t)$ are negligible. Using this relation, the scale parameter 255 can be interpreted as the wavelet width in time units (here in days) (table 1).

256 For a value of $a=0.1$, all daily changes were analyzed in detail by the wavelet. For a value of $a=6$, the time series was smoothed and we obtained the main tendency of changes through about 36 days. Higher values of the scale parameter $a$ smoothed all time variations of the series, with no interest from an epidemiological point of view. Hence, for a study of about 800 days, the useful range of the parameter $a$ was between 0.1 and 6.0. In the context of longer time series and/or slower disease transmission, larger values of the scale parameter may be more suitable.

\subsection{Wavelet spectrum and cross-wavelet spectrum}

264 Real wavelet transform can highlight the local characteristics of a time series, in particular its rapid changes and burst amplitudes. Let us consider the epidemiological series $s(t)$ and the

266 wavelet coefficients $W(a, b)$. The local power spectrum is defined as:

$267 \quad S(a, b)=|W(a, b)|^{2}$

268 For a given scale, that is a given resolution, the spectrum varies as a function of the translation

269 parameter. These variations exhibit the spectral peaks of the original time series. The 270 magnitude of each peak represents the epidemic series dynamic: the more rapid the variation, 271 the higher the spectral peak. A nonzero constant time series has a null spectrum. In epidemic 272 analysis, it is of high interest to note the increase or decrease of case toll, but, according to 
273 equation (17), spectra have only positive values. For this interpretability reason, we modified

274 the definition of the spectra as follows:

$275 S^{*}(a, b)=\operatorname{sign}(W(a, b)) \times|W(a, b)|^{2}$

276 Thus, an increase of the time series at time $t$ was characterized by a positive value of $S^{*}(a, b)$

277 at $b=t$ (the faster the increase, the higher value the spectrum). Conversely, a decrease of the

278 time series at $t$ was characterized by a negative value of $S^{*}(a, b)$ at $b=t$ (the faster the

279 decrease, the lower value the spectrum). Absence of change in time series provided a null

280 spectrum, whatever the cholera case toll.

281 As rainfall is an important driver of cholera epidemic, we also analyzed rainfall time series, and

282 compared epidemic wavelet spectra and rainfall wavelet spectra. The wavelet coherence,

283 measuring the cross-correlation between two time series, has been defined for complex

284 wavelets [17,22]. In the case of real wavelets, such as the GCP wavelet, the wavelet coherence

285 is equal to 1 whatever times and scales, and therefore cannot be used. Therefore, we used the

286 cross-wavelet spectrum defined by the following equation:

$C W(a, b)=\frac{W_{c}(a, b) \times W_{r}(a, b)}{\max \left|W_{c}(a, b)\right| \times \max \left|W_{r}(a, b)\right|}$

288 where $W_{c}(a, b)$ and $W_{r}(a, b)$ are the cholera epidemic coefficients and rainfall coefficients,

289 respectively. A denominator was added to the classical cross-wavelet spectrum definition in

290 order to re-scale both spectra at a same magnitude.

291 Cross-wavelet spectra reveal the underlying relationship between two time series. In our study

292 it highlighted the interactions between rainfall and cholera epidemic. Furthermore, to provide 293 a thorough description of these relationships, a lag parameter between rainfall and cholera cases

294 was introduced in the cross-wavelet spectrum. By analyzing cholera cases at a time $t$ and 295 rainfall at a time $t$-lag, the lag parameter varied from 0 to a value specified when a cross-wavelet 296 maximum was reached. These lags indicated the number of days between rainfall and its 297 potential consequence on the cholera case toll. In order to evaluate cross-wavelet at different 
lags and times, lagograms were developed that provided cross-wavelet spectra varying through

299 lags and times.

300 Program development and data analysis were conducted using Matlab 7.0.1 (The Mathworks

301 Inc., Natick, MA, USA).

\subsection{Comparison to a change point analysis}

303 Results obtained by the developed wavelet analysis of the cholera case toll have been compared

304 to a change point analysis, applying the Segment Neighbourhood (SN) method [37]. This

method is considered as an exact one for change point detection, evaluating all possible

segmentations to a maximum number of change points. This maximal number of change points,

14 , have been chosen higher than the number of spectral peaks determined by the wavelet analysis. A Poisson distribution and a penalty value of $1.5 \times \log (n)$ have been applied. Change

309 detection in mean and variance was performed using $\mathrm{R} \circledR \mathrm{v} 3.0 .1$ (The $\mathrm{R}$ Foundation for

310 Statistical Computing, Vienna, Austria) with the changepoint package, provided by Killick et

311 al. [38].

\section{$312 \quad 3$ Application}

\section{$313 \quad 3.1 \quad$ Cholera time series analysis}

314 During the study period, the daily cholera case toll was prospectively collected by Haitian

315 Departmental Health Directorates. Departmental databases were sent to the Haitian Directorate 316 of Epidemiology, Laboratory and Research (DELR), where data were gathered and analyzed 317 after quality control [24,25]. In this study, we did not include personal data but only the national 318 daily toll of cholera cases. Its time series was analyzed from September 01, 2010 to November 31920,2012 (812 days). In order to eliminate edge effects, we excluded from the results the 10 320 first days and the 10 last days. Calculation of the wavelet coefficients (using the normalized 321 wavelet and scanning the whole period) provided a spectral value for each value of the 322 translation parameter $b$, from $t=1$ to 812 days, and for each value of the scale parameter $a$. 
The time series and spectra (at different values of the scale parameter $a$ ) are shown in figure 2.

324 The scalogram represents spectrum values according to the scale and the translation parameters

325 (figure $2 \mathrm{~B}$ ).

326 The spectral analysis (figures 2C and 2D) highlighted 4 spectrum peaks corresponding to 4 327 bursts of the epidemic that were related to different epidemic mechanisms. The first spectrum 328 peak corresponded to the explosive start at the lower Artibonite valley on October 19, 2010 329 (just after its initiation in the commune of Mirebalais [24]). This high spectrum peak had an 330 impact towards the whole study period, as it remained high at large values of the scale 331 parameter. The second spectrum peak, with a lower magnitude, corresponded to the epidemic 332 spread out of the Artibonite valley (around November 07, 2010). The third spectrum peak 333 corresponded to worsening in cholera incidence concomitant to riots in Port-au-Prince 334 following the first round of presidential elections in early December 2010. This spectrum peak 335 was followed by negative spectrum values mainly shown at large scale (figure 2D), 336 corresponding to the slow decrease of the epidemic. After a lull phase, the fourth spectrum 337 peak corresponded to the rainy season (May 2011). This peak was related to an outbreak that 338 showed a profile different from the first ones: its dynamic was more progressive [25]. It was 339 highlighted by the lower magnitude but larger area under the curve of the fourth spectrum peak 340 when considering large values of the scale parameter. The magnitude of these 4 spectrum peaks 341 still remained even if using a larger scale parameter $(a=8)$. Following these four peaks, the 342 magnitude of the spectrum decreased to undetectable spectrum values which can be interpreted 343 as a slow trend towards epidemic stability as time passed. The particularly high magnitude of 344 the first spectrum peak explained the major part of the variability of the time series. Subsequent 345 small variations of the cholera case toll could thus not be seen.

346 The change point analysis (figure 3) detected a high number of change points $(\mathrm{q}=14)$ : the 347 epidemic onset and the 2 following peaks have been detected, as well as the increases in June 
2011 and May 2012. But the decrease at the end of October 2010 has not been detected. The

349 decrease between December 2010 and March 2011 has been split in 3 segments, as well as the decrease from June to August 2011.

\subsection{Rainfall time series analysis}

352 Daily accumulated rainfall data were obtained through the NASA Goddard Earth Sciences, at 353 the national level. These observations were derived from the Tropical Rainfall Measuring 354 Mission (TMPA-RT 3B42RT); for more details see 355 http://disc.sci.gsfc.nasa.gov/giovanni/overview/index.html (access: September, 2013). 356 Rainfall time series and spectra (at different values of the scale parameter) are presented in 357 figure 4 . The scalogram represents spectrum values according to the scale and translation 358 parameters (figure 4B). The spectral analysis of rainfall exhibited two major spectrum peaks 359 related to hurricane Tomas (November 05, 2010) and hurricane Sandy (October 24, 2012), 360 respectively. Peaks detected in the meanwhile, especially for large values of the scale 361 parameter, corresponded to the 2011 and 2012 rainy seasons. No high rainfall had been 362 exhibited before the first cholera case burst.

\section{$363 \quad 3.3 \quad$ Cross-wavelet spectrum}

364 The cross-wavelet spectra calculated with a null time lag are presented in figure 5. They showed 365 a globally low relationship between cholera cases and rainfall, except for the epidemic on May 3662011 at large values of the scale parameter (figure 5C), what field epidemiologists also reported.

367 The negative values of the cross-wavelet spectra at the beginning of the studied period can be 368 interpreted as a decoupling of the 2 time series. The relationship between the two time series 369 had thus to be studied at different time lags. Indeed, a lag around 3-4 days, between rainfall 370 and cholera case toll is highlighted by the lagogram, for scale parameter $a=1.7$ (10 days)

371 (figure 6). Maximum of the cross-wavelet spectra occurred for a 3 day lag at $b=58$ days, 372 around November 08, 2010 (second cholera case burst), 3 days after hurricane Tomas reached 
373 Haiti (table 2). Concerning the third and fourth cholera burst, a small amount of rainfall was

374 reported 5-6 days before the third cholera burst (around December 15, 2010). The rainy season

375 began around May 20, 2011, 5-6 days before the onset of the fourth cholera case burst. It is

376 noticeable that no relationship had been exhibited between the decrease of both the cholera case

377 toll and rainfall. Furthermore, high cross-spectra were followed by very low (negative) cross-

378 spectra (figure 6), i.e. the transient coupling of the two series were systematically followed by

379 their decoupling. This can be interpreted as a booster effect of rainfall on an ongoing cholera

380 epidemic, after which transmission is neither further accelerated by persisting rainfall, nor

381 immediately stopped with rain cessation.

\section{Discussion}

383 Applied to non-periodic non-stationary epidemic time series, the wavelet transform, using the

384 GCP wavelet, highlighted the main features of the cholera epidemic in Haiti and its relationship

385 with rainfall. The wavelet spectra provided timely accurate information about speed and 386 intensity of epidemic variations. These variations could not be easily evaluated by only 387 observing the case toll time series, because of the violence of the first bursts due to the particular context of this epidemic. Wavelet transform also highlighted slower events such as the epidemic decreases after the third and the fourth bursts, in a multi-resolution approach. As spectrum calculation was based on a convolution between the time series and the wavelet, each

391 spectrum was calculated considering the whole time series, i.e. the whole epidemic dynamic.

392 Using a continuous scale parameter, the wavelet transform exhibited the epidemic variations at 393 different time scale, as showed by scalograms. This was of high interest for field 394 epidemiologists, as it led to more accurately assess the epidemic evolutions. Three types of 395 spectral peaks have been observed: i) peaks only observed at narrow scale values, which were 396 very precisely localized in time; ii) peaks only observed at large scale values, which exhibited slower epidemic dynamics through larger time intervals; iii) peaks observed both at narrow and 
large scale values, which not only exhibited a time localized variation, but also a variation that

399 influenced a large time interval. Persistence of high wavelet spectra along a large range of scale

400 parameter values highlighted the magnitude of the first burst, and its impact on the whole

401 epidemic.

402 The formal relationship between wavelet approach and statistical analysis has been made only

403 for stationary time series under the assumption of dyadic sample size $[18,39]$. The spectrum

404 expectation can be interpreted as the variance of the time series although this has not been yet

405 formally demonstrated for non-stationary time series and non-dyadic sample size.

406 Nevertheless, for a given scale parameter $a$, the spectrum expectation can be interpreted as a

407 variability measure of the time series. This has practical implication in interpreting the spectra.

408 Indeed, the relative importance of a variation at time $t$, relatively to the whole time series, can

409 be measured by the relative magnitude of the spectrum value for $b=t$, relatively to the

410 spectrum expectation. From an epidemiological point of view, the case spectra need a separate

411 evaluation for the second year of the epidemic. Indeed, as relative measures, spectra of the

412 second year are much smaller than the 4 peaks of the first year. The second year counted for

413 about 102,582 cases (vs 493,069 cases during the first year), and cholera outbreaks of lower

414 magnitude appeared during the rainy seasons (around October 2011 and May 2012).

415 In our context, the choice of the GCP wavelet has been mainly led by the non-stationary feature

416 and the non-periodicity of the case and rainfall time series, and by the research of precise time

417 localization of their features. This wavelet with a null mean and no period presents one positive

418 and one negative part only, both with equal areas under the curve. With its simple analytical

419 form, the GCP wavelet led us to quantify the rapid changes of the cholera case toll. The

420 parameter $c$ (see equation 11), which remained fixed in this study, can be used to tune the speed

421 of the steady-state wavelet return. 
422 Spectral analysis, through wavelet transform, can be considered as an alternative to the classic

423 change point analysis [37,38]. Wavelet approaches provide different information about both

424 time and frequency domains. It allows to extract the main features explaining the behavior

425 underlying a time series, facilitate aberration detection and epidemiologic analysis. In our

426 context, a few features (timely localized or periodic) explaining a time series can be useful from

427 an epidemiological point of view, as well as studying relationships between two time series.

428 Classical change point analyses are considered as linear approaches and do not allow

429 relationship analysis. As a nonparametric non-linear approach, wavelet analysis is able to adapt

430 to local and global features, which is not possible with classical linear approaches [10]. GCP

431 wavelet approach could be particularly interesting to analyze disease surveillance time series.

\section{Acknowledgements}

434 The authors acknowledge Anestis Antoniadis for his review of this manuscript and Louis

435 Gaudart for his helpful comments on wavelet theory, particularly on the GCP wavelet. The 436 authors also acknowledge Dr Roc Magloire, from the MSPP, Haiti.

437 Funding statement: The epidemiological field study was supported by the French Embassy in 438 Port-au-Prince (Haiti). Dr. Jean Gaudart was also supported by the ADEREM association for 439 biological and medical research development (Association pour le Développement des 440 Recherches biologiques et Médicales, http://www.aderem.fr). The funders played no role in 441 study design, data collection and analysis, the decision to publish, or preparation of the 442 manuscript.

443 Competing interests: The authors declare that no competing interests exist. 


\section{Data accessibility}

445 Cholera case time series is available through the website of the Ministry of Health of Haiti 446 (http://mspp.gouv.ht/newsite/). Rainfall time series is available through the NASA Goddard 447 Earth Sciences website (http://disc.sci.gsfc.nasa.gov/giovanni/overview/index.html).

\section{References}

450 1. Maraun D, Kurths J, Holschneider M. 2007 Non stationary Gaussian processes in wavelet 451 domain: estimation, and significance testing. Phys. Rev. E 75, 016707.

452 2. Shumway RH, Stoffer D. 2011 Times series analysis and its application (with R examples),

453 3rd edition. Springer texts in statistics. London, UK: Springer.

454 3. Loboda NS, Glushkov AV, Khokhlov VN, Lovett L. 2006 Using non-decimated wavelet 455 decomposition to analyse time variations of North Atlantic Oscillation, eddy kinetic energy, 456 and Ukrainian precipitation. J. Hydrol. 322, 14-24.

457 4. Eriksson C, Omstedt A, Overland JE, Percival DB, Mofjeld HO. 2007 Characterizing the 458 European sub-arctic winter climate since 1500 using ice, temperature, and atmospheric 459 circulation time series. J. Climate 20, 5316-5334.

460 5. Ménard, F., Marsac, F., Bellier, E. and Cazelles, B. 2007 Climatic oscillations and tuna 461 catch rates in the Indian Ocean: a wavelet approach to time series analysis. Fish. Oceanogr. 462 16, 95-104.

463 6. Prokoph A, El Bilali H. 2008 Cross-Wavelet Analysis: a tool for detection of relationship 464 between paleoclimate proxy records. Math. Geosci. 40, 575-586.

465 7. Sanderson J, Fryzlewicz P, Jones MW. 2010 Estimating linear dependence between 466 nonstationary time series using the locally stationary wavelet model. Biometrika 97, 435-466.

467 8. Mallat SG. 1989 Multifrequency channel decompositions of images and wavelet models. 468 IEEE T. Audio Speech Lang. Process. 37, 2091-2110. 
9. Saab R, McKeown MJ, Myers LJ, Abu-Gharbieh R. 2005 A wavelet based approach for the detection of coupling in EEG signals. Proceedings of the 2nd International IEEE EMBS functional linear models: an application to measurement error-corrected distributed lag models. Biostatistics 11, 432-452. spectral analysis of epidemiological time-series with wavelets. J. R. Soc. Interface 4, 625-636. synchrony of cholera epidemics in West Africa. BMC Infect. Dis. 7, 20. 20120814.

18. Nason GP. 2008 Wavelet methods in statistics with R, 1rst edition. Use R!. New York,

$490 \quad$ NY: Springer.

491 19. Barichivich J, Sauchyn D, Lara A. 2009 Climate signals in high elevation tree-rings from

492 the semiarid Andes of north-central Chile: Responses to regional and large-scale variability.

493 Palaeogeogr. Palaeocl. 281, 320-333. 
20. Chui CK. 1992 An introduction to wavelets, 1rst edition. Boston, MA: Academic Press.

21. Morlet J, Arens G, Fourgeau E, Giard D. 1982 Wave propagation and sampling theory, Part II. Geophysics 47, 222-236.

22. Grinsted A, Moore JC, Jevrejeva S. 2004 Application of the cross wavelet transform and wavelet coherence to geophysical time series. Nonlinear Proc. Geoph. 11, 561-566.

23. Gabor D. 1946 Theory of communication. J. I. E. E. 93, 429-457.

24. Piarroux R, Barrais R, Faucher B, Haus R, Piarroux M, Gaudart J, Magloire R, Raoult D. 2011 Understanding the cholera epidemic, Haiti. Emerg. Infect. Dis. 17, 1161-1168.

25. Gaudart J, Rebaudet S, Barrais R, Boncy J, Faucher B, Piarroux M, Magloire R, Thimothe G, Piarroux R. 2013 Spatio-Temporal Dynamics of Cholera during the First Year of the Epidemic in Haiti. PLoS Neglect. Trop. D. 7, e2145.

26. Mallat SG. 1989 A theory for multiresolution signal decomposition: the wavelet representation. IEEE T. Pattern Anal. 11, 674-693.

27. Gaudart L, Crebassa J, Petrakian JP. 1992 Comparative analysis of wavelet models and vision. J. Optics 23, 101-106.

28. Gaudart L, Crebassa J, Petrakian JP. 1993 Wavelet transform in human visual channels. Appl. Optics 32, 4119-4127.

29. Grossmann A, Morlet J. 1984 Decomposition of Hardy functions into square integrable wavelets of constant shape. SIAM J. Math. Anal. 15, 723-736.

30. Daubechies I. 1988 Orthonormal bases of compactly supported wavelets. Commun. Pur. Appl. Math. 41, 909-996.

31. Daubechies I. 1990 The wavelet transform, time-frequency localization and signal analysis. IEEE T. Inform. Theory 36, 961-1005.

32. Cazelles B, Chavez M, Berteaux D, Ménard F, Vik JO, Jenouvrier S, Stenseth NC. 2008 Oecologia 156, 287-305. 
519 33. Broutin H, Philipon S, De Magny GC, Courel MF, Sultan B, Guégan JF. 2007

520 Comparative study of meningitis dynamics across nine African countries: a global

521 perspective. Int. J. Health Geogr. 6, 29.

522 34. Yang L, Wong CM, Lau EHY, Chan KP, Ou CQ, Peireis JSM. 2008 Synchrony of

523 clinical and laboratory surveillance for influenza in Hong Kong. PLoS One 3, e1399.

524 35. Thai KTD, Cazelles B, Nguyen NV, Vo LT, Boni MF, Farrar J, Simmons CP, Van Dorn

525 HR, De Vries PJ. 2010 Dengue dynamics in Binh Thuan province, Southern Vietnam:

526 periodicity, synchronicity, and climate variability. PLoS Neglect. Trop. D. 4, e747.

527 36. Hsu L, Self SG, Grove D, Randolph T, Wang K, Delrow JJ, Loo L, Porter P. 2005

528 Denoising array-based comparative genomic hybridization data using wavelets. Biostatistics

$529 \quad 6,211-226$.

530 37. Auger IE, Lawrence CE. 1989 Algorithms for the optimal identification of segment

531 neighborhoods. B. Math. Biol. 51, 39-54.

532 38. Killick R, Fearnhead P, Eckley IA. 2012 Optimal detection of changepoints with a linear

533 computational cost. J. Am. Stat. Assoc. 107, 1590-1598.

534 39. Antoniadis A. 2007 Wavelet method in statistics: some recent developments and their

535 applications. Stat.Surv. 1, 16-55. 


\section{Figures}
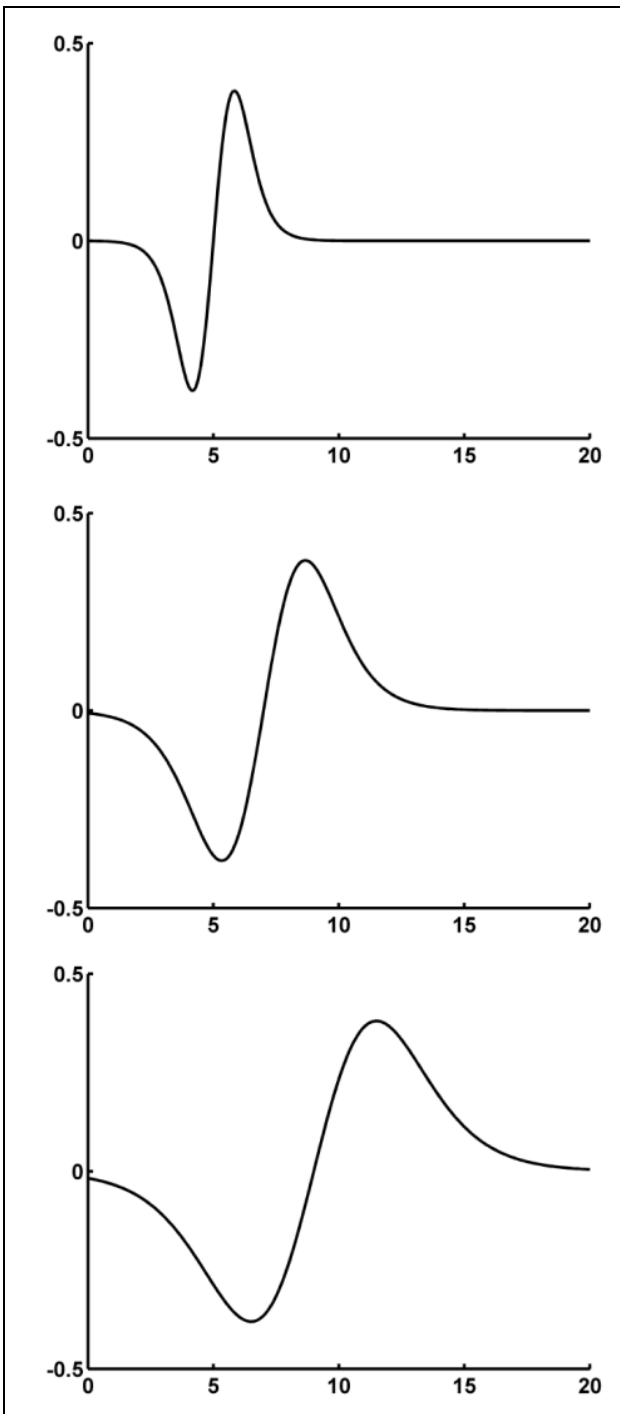

539 Figure 1. Wavelet variations as a function of time. Non normalized wavelets $\Psi(t ; a, b)$ are

540 plotted for different values of scale and translation parameters. The $\mathrm{x}$-axis represents the time

541 (days). The y-unit is arbitrary. Upper panel: $a=1, b=5$; central panel: $a=2, b=7$; lower

542 panel: $a=3, b=9$. 


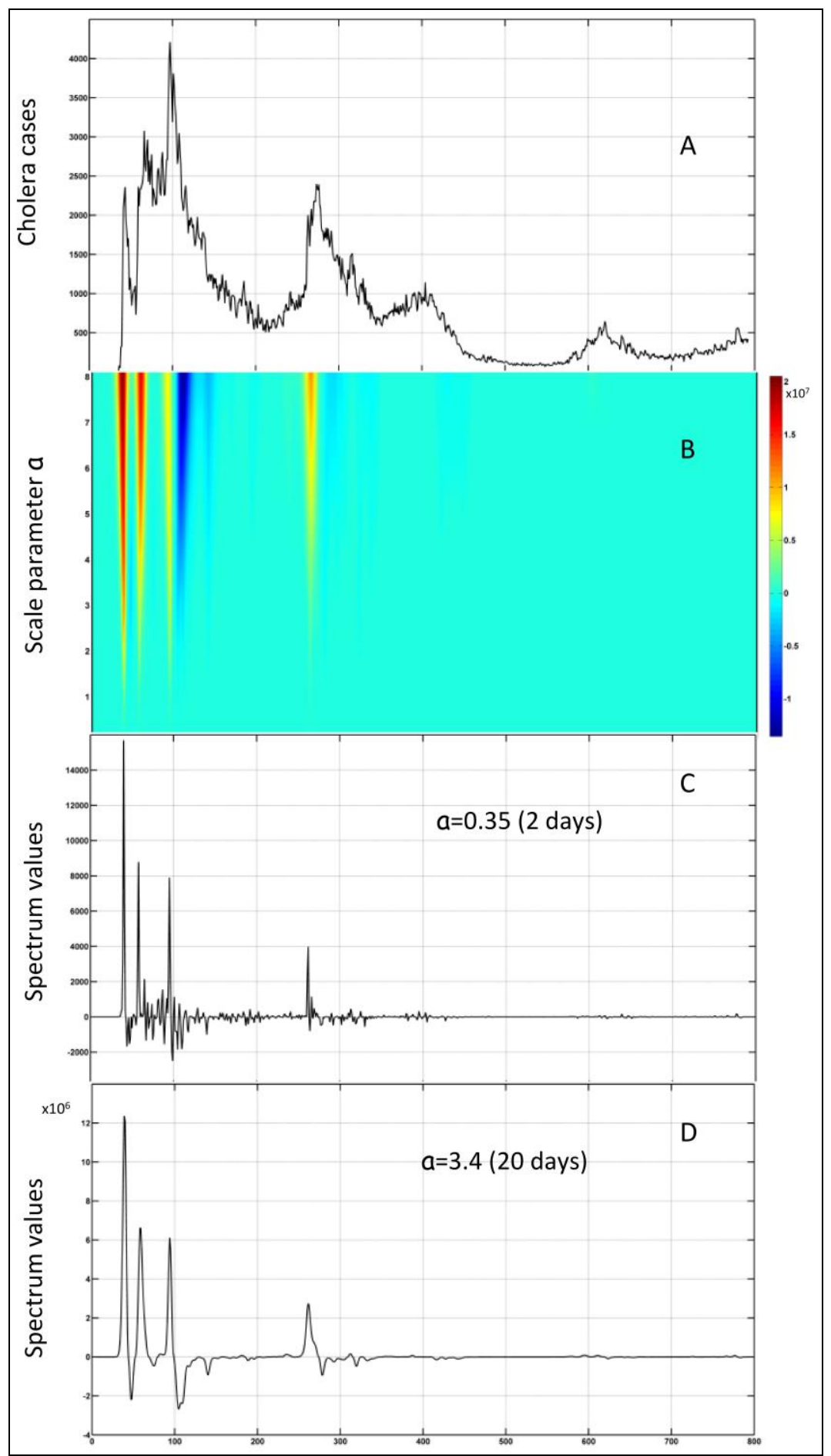

545 Figure 2. Cholera time series and wavelet transform. The x-axes represent the time (days)

546 from September 10, 2010 to November 10, 2012. A) Daily reported cholera case toll; B)

547 Cholera case toll scalogram ( $a$ from 0.1 to 8 ). The coloured scale represents the values of the

548 spectra $S^{*}(a, b)$ (arbitrary units). High values are shown in dark red and low values 
549 (negative) in dark blue; C) Spectra for a narrow wavelet width: $a=0.35$; D) Spectra for a large 550 wavelet width: $a=3.4$.

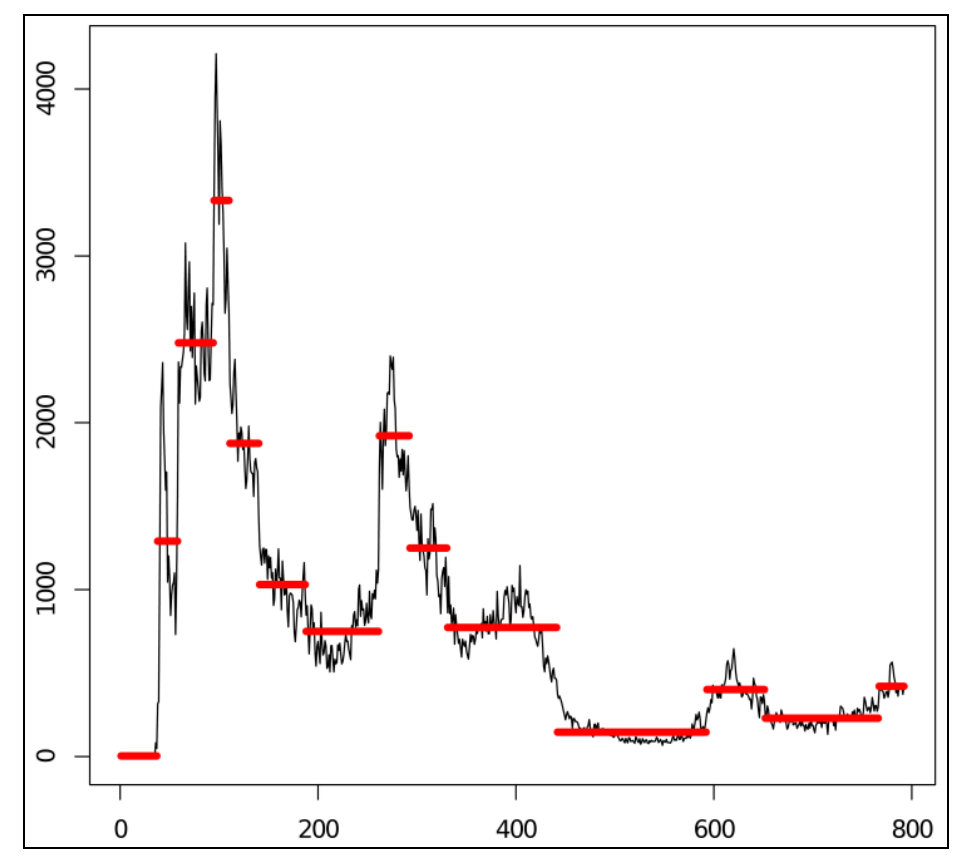

553 Figure 3. Cholera time series and Change Point Analysis. The $\mathrm{x}$-axis represents the time

554 (days) from September 10, 2010 to November 10, 2012. The y-axis represents the Cholera

555 case toll. The red horizontal lines identify the changes in the cholera case mean. 


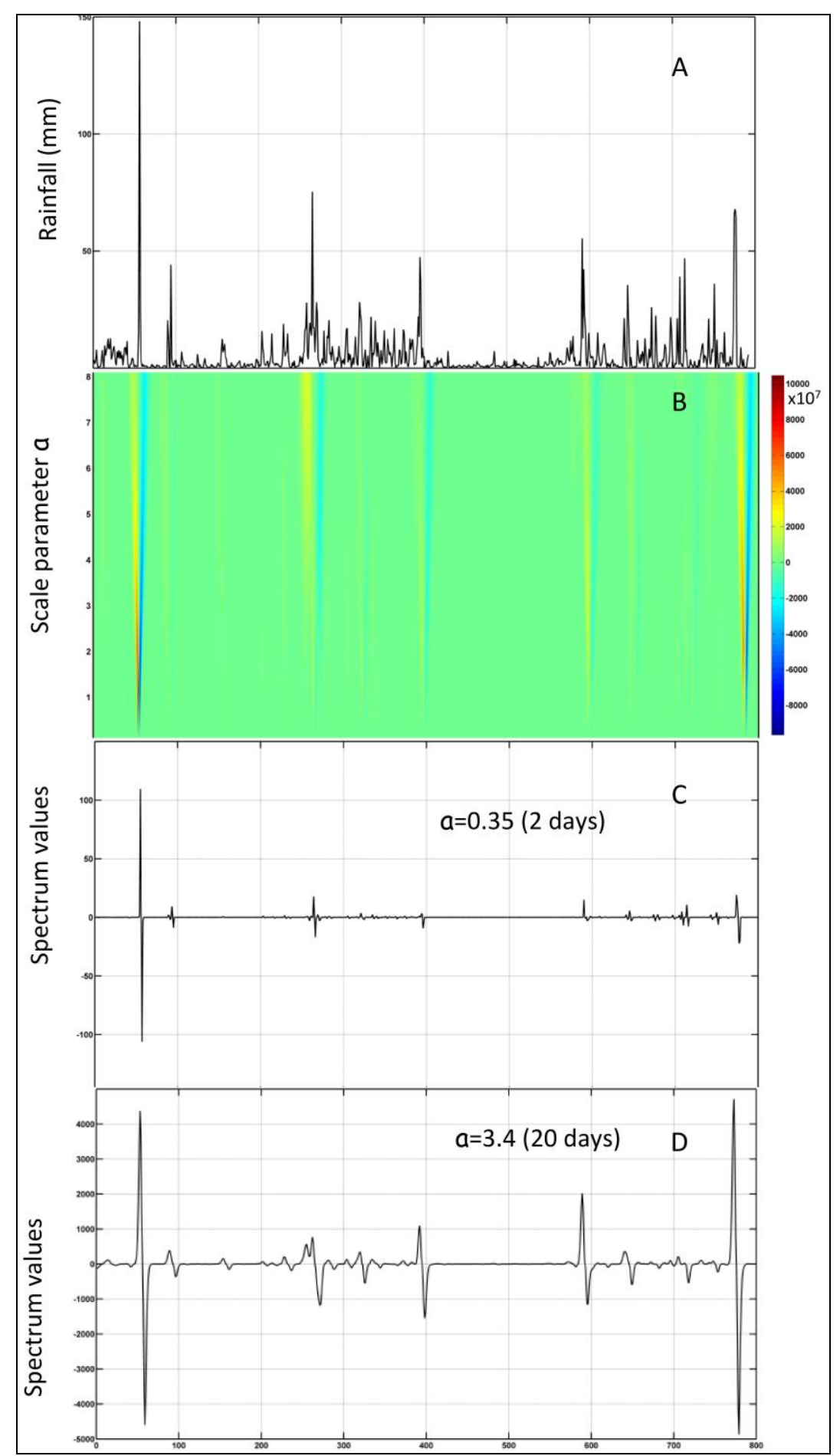

558 Figure 4. Rainfall time series and wavelet transform. The $\mathrm{x}$-axes represent the time (days)

559 from September 10, 2010 to November 10, 2012. A) Daily reported rainfall; B) Rainfall

560 scalogram ( $a$ from 0.1 to 8 ). The coloured scale represents the values of the spectra $S^{*}(a, b)$

561 (arbitrary units). High values are shown in dark red and low values (negative) in dark blue; 
C) Spectra for a narrow wavelet width: $a=0.35$; D) Spectra for a large wavelet width: $a=$

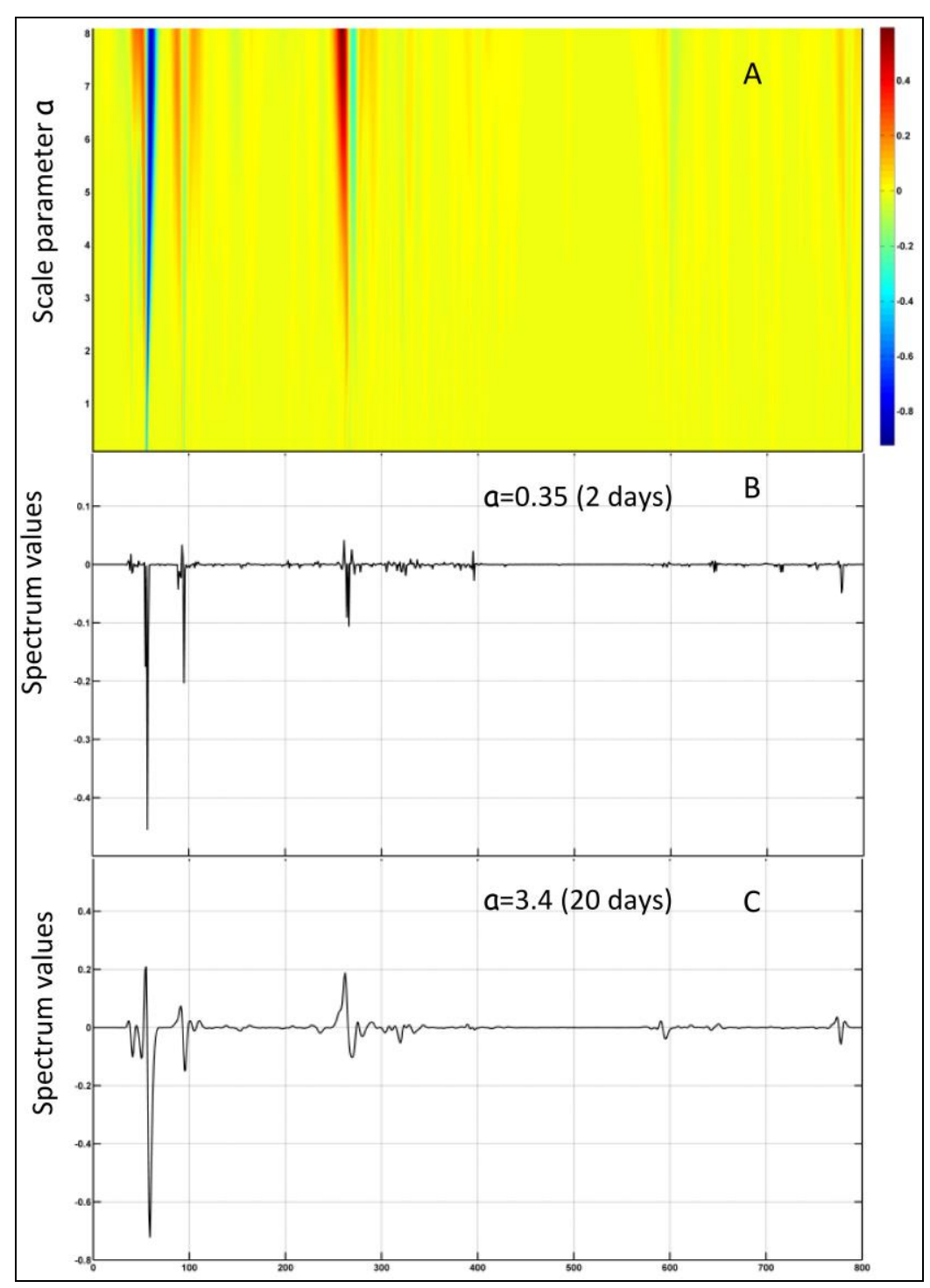

566 Figure 5. Cholera case toll and rainfall cross-wavelet spectra, lag=0. The $\mathrm{x}$-axes represent

567 the time (days) from September 10, 2010 to November 10, 2012. A) Cross-wavelet

568 scalogram ( $a$ from 0.1 to 8 ). The coloured scale represents the values of the cross-spectra

$569 C W(a, b)$ (arbitrary units). High values are shown in dark red and low values (negative) in

570 dark blue; B) Cross-spectra for a narrow wavelet width: $a=0.35$; C) Cross-spectra for a

$571 \quad$ large wavelet width: $a=3.4$. 


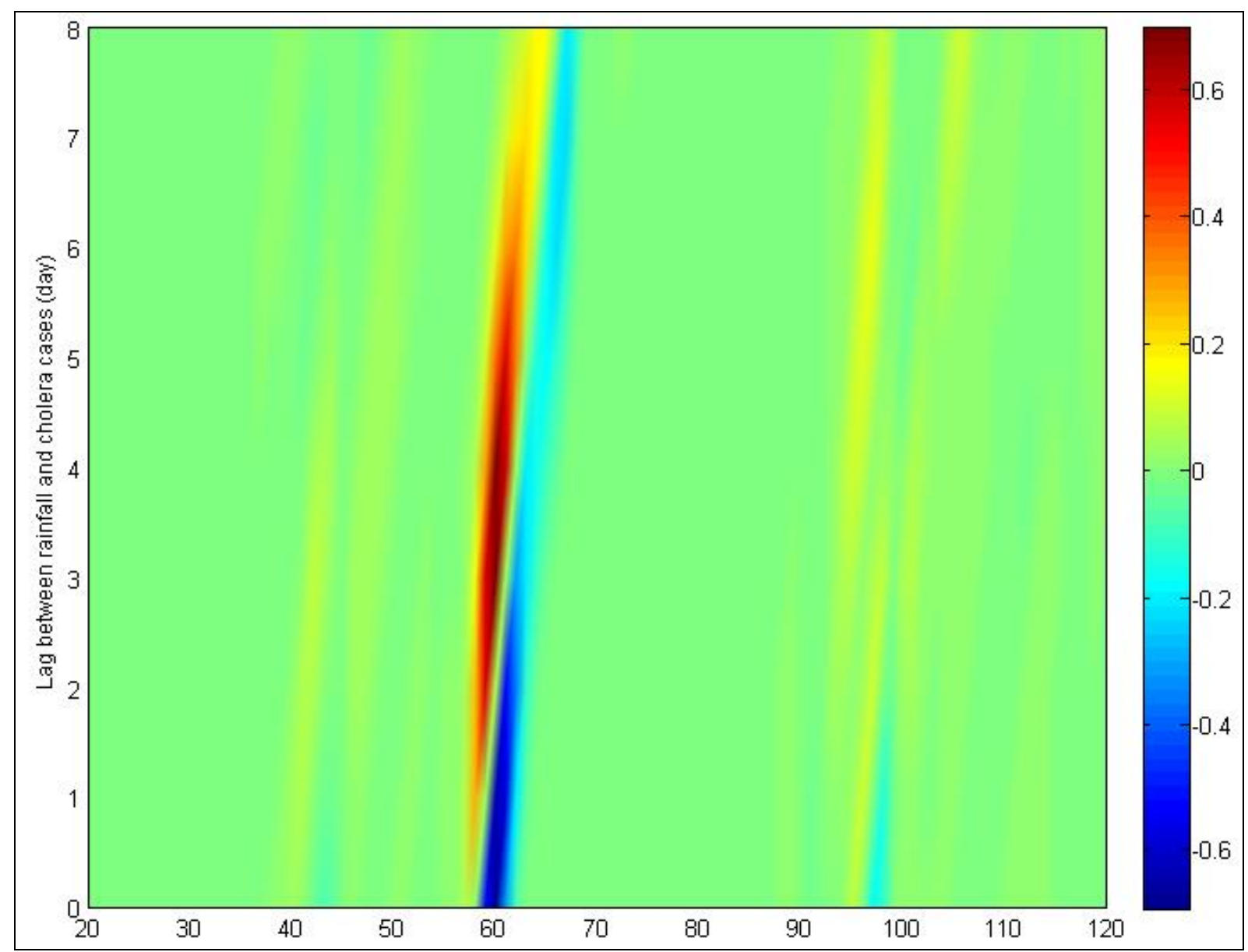

573 Figure 6. Cross-wavelet lagogram: cholera case toll and rainfall cross-wavelet spectra, with

574 various lags; $a=1.7$ (10 days). The x-axis represents the time (days) from October 01, 2010

to January 08, 2011 (100 days). The y-axis represents the value of the lag between rainfall and cholera case toll, ranging from 0 to 8 days. The coloured scale represents the values of the cross-spectra $C W(a, b)$ (arbitrary units). High values are shown in dark red and low values (negative) in dark blue. 


\section{Tables}

585

586 Table 1: Relationship between the scale parameter $a$ and the observed time width $L$ of the

587 wavelet (days).

\begin{tabular}{ccccccccc}
\hline$a$ & 0.1 & 0.5 & 1.0 & 2.0 & 3.0 & 4.0 & 6.0 & 8.0 \\
& & & & & & & & \\
\hline Width $L$ (days) & 1 & 3 & 6 & 12 & 18 & 24 & 36 & 47
\end{tabular}

590 Table 2: Cross-wavelet spectrum maxima. Maximal values of cross-spectra are presented

591 according to the lag between cholera case toll and rainfall, for $a=1.7$ (10 days wavelet width).

592 The time column represents the related date of the cholera case toll.

Lag (days) Cross-spectrum maxima (a.u. ${ }^{*}$ Time

(days from Sept. 10, 2010)

\begin{tabular}{llc}
\hline 1 & 0.2684 & 56 \\
2 & 0.5601 & 57 \\
3 & 0.6956 & $58^{* * *}$ \\
4 & 0.6766 & 58 \\
5 & 0.5513 & 59 \\
6 & 0.3377 & 60 \\
7 & 0.2042 & 62 \\
8 & 0.1729 & 63 \\
\hline
\end{tabular}

